\section{Unequal equals}

Nature 583, 211-214 (2020)

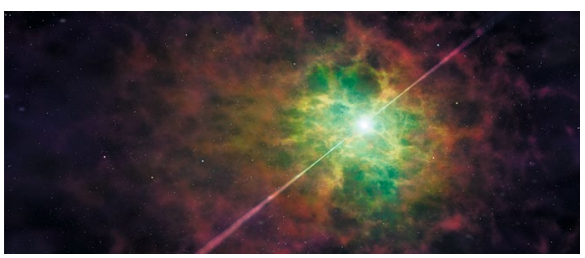

Credit: Mark Garlick / Science Photo Library / Getty

The late-time electromagnetic emission of the kilonova associated with the gravitational wave signal GW170817 from a binary neutron star merger is at odds with theoretical expectations. A binary system with an asymmetric mass ratio is one potential explanation. However, the known binary neutron star systems that are expected to merge within billions of years have similar component masses.

Robert Ferdman and colleagues have now identified the most asymmetric double neutron star system observed to date. They precisely measured the mass ratio of the pulsar PSR J1913+1102 and estimated its coalescence time to be around 470 million years. A population synthesis analysis of the nine merging binary neutron star systems with known masses showed that several per cent of all merging binaries have similarly asymmetric mass ratios. This implies the existence of a significant population of asymmetric binary neutron star systems, which could explain the anomalous properties of GW170817, and is expected to lead to future detections of bright kilonovae.

https://doi.org/10.1038/s41567-020-1014-6
NEUTRON STAR MERGERS

\section{Test your theory}

Phys. Rev. Lett. (in the press); preprint at https:// arxiv.org/abs/2003.00349

Quantum theory has been put to many tests and it has passed them all, but it is not the only possible theory to explain the physical world. Unlike classical theories, quantum mechanics does not rest on clear physical principles, and it is therefore difficult to identify it as the one correct theory. Mirjam Weilenmann and Roger Colbeck have now suggested an alternative approach to single out quantum mechanics: they proposed to extend the concept of self-testing quantum states to the theory itself.

When two particles are entangled, separate measurements of each particle are correlated in a way that cannot be reproduced classically. Observing these correlations provides a 'self-test' that shows entanglement is present without the need to know any details of the measurement.

Weilenmann and Colbeck proposed searching for self-tests that are satisfied by quantum mechanics but exclude alternative theories. Defining quantum theory via self-tests could provide a more intuitive basis for quantum mechanics, and self-tests could be used to experimentally confirm the validity of quantum theory.

https://doi.org/10.1038/s41567-020-1013-7

\section{QUANTUM METROLOGY}

\section{Spins sense fields}

Phys. Rev. X 10, 031003 (2020)

The electron spins localized at colour centres in diamond can be used as

\section{QUANTUM MECHANICS}

\section{TOPOLOGICAL PUMPING}

\section{Lost and found}

A rotating screw pump lifts water along its axis, although the position of the screw itself is fixed inside its housing. Similarly, an adiabatic modulation of a periodic potential can move particles from one potential minimum to the next. However, this so-called Thouless pumping loses its quantization in the presence of losses or when the drive is not adiabatic. Zlata Fedorova and colleagues have now shown that the quantization can be recovered if the dissipation is also periodically modulated.

When they incorporated this modulation in their theoretical modelling, counter-propagating modes in a lossy system could no longer hybridize, which restored the quantization - even if the modulation of the driving potential was non-adiabatic. Fedorova and colleagues realized their model using coupled sinusoidal plasmonic waveguides, for which the periodically varying losses were achieved by modulating the cross-section that controls the mode confinement. Despite the optical demonstration, this dissipative Floquet engineering can be applied to a wider class of periodically driven systems.

https://doi.org/10.1038/s41567-020-1012-8 magnetic field sensors, in part because the almost-atomic size of the defect allows them to be packed at a high density that increases sensitivity. However, there is a limit to this optimization because the interactions between closely spaced spins cause decoherence of the ensemble.

Hengyun Zhou and collaborators have now found a way to push the sensitivity past this fundamental limit. They applied a carefully chosen sequence of laser pulses to the spin system, which extended the coherence time beyond the limit set by the interactions. The sensitivity for the magnetic field could be enhanced by $40 \%$, so that weaker fields can be detected.

This work shows how delicatelycontrolled high-density spin ensembles can used in quantum metrology and suggests that they can also be employed as sensors of observables other than magnetic fields in the future.

https://doi.org/10.1038/s41567-020-1011-9

\section{QUANTUM GASES}

\section{An ultracold junction} Science 369, 84-91 (2020)

Two superconductors weakly coupled through an insulating potential barrier form a Josephson junction. Electrons on each side of the barrier can be described by a macroscopic wavefunction, and the phase difference between them leads to a current flowing across the junction - known as the Josephson effect. Now, Woo Jin Kwon and co-workers, as well as Niclas Luick and co-workers, have observed the same effect in charge-neutral systems made of ultracold ${ }^{6} \mathrm{Li}$ atoms.

Like electrons, the atoms in these experiments are fermions, and can form pairs and condense in the same quantum state at sufficiently low temperature. An optical barrier inserted in the centre turned the system into a junction. A current across the barrier was induced either by imposing an initial phase difference between the two parts or by directly moving the optical barrier with respect to the atoms. Both groups observed a sinusoidal current-phase relation - a distinctive feature of the Josephson effect by monitoring the subsequent evolution of the atom number and of the phase of the wavefunction. 